\title{
Solar Flare Intermittency and the Earth's Temperature Anomalies
}

\author{
Nicola Scafetta ${ }^{1,2}$ and Bruce J. West ${ }^{1,2,3}$ \\ ${ }^{1}$ Pratt School EE Dept., Duke University, P.O. Box 90291, Durham, NC 27708 \\ 2 Physics Department, Duke University, Durham, NC 27708 and \\ 3 Mathematics Division, Army Research Office, Research Triangle Park, NC 27709.
}

(Dated: June 12, 2018)

\begin{abstract}
We argue that earth's short-term temperature anomalies and the solar flare intermittency are linked. The analysis is based upon the study of the scaling of both the spreading and the entropy of the diffusion generated by the fluctuations of the temperature time series. The joint use of these two methods evidences the presence of a Lévy component in the temporal persistence of the temperature data sets that corresponds to the one that would be induced by the solar flare intermittency. The mean monthly temperature datasets cover the period from 1856 to 2002.

PACS numbers: $95.75 . \mathrm{Wx}, 05.40 . \mathrm{Fb}, 05.45 . \mathrm{Tp}, 47.27 . \mathrm{Nz}$
\end{abstract}

The historical recognition that the sun warms the earth has suggested a direct connection between the average global temperature and solar activity. Consequently any significant changes in solar activity should result in equivalent changes in the earth's global temperature. The literature on the solar influence on the earth's temperature is quite extensive [1, 2, [3], indicating the importance of the problem and that there are many issues that require further investigation. Herein we address the relation between the statistics of solar flare activity and the fluctuations in the earth's global temperature.

The dynamics of the sun's surface is turbulent, as is evidenced by changes in solar flare activity, with 11 or 22 year solar cycles [2, 3] and strong erratic fluctuations associated with solar flare intermittency $[3,4,5,6$, 6 . Solar irradiance changes in accordance with the frequency of solar flares because each flare releases more energy than the background irradiance [3]. This time variation in the frequency of solar flares induces a similar pseudoperiodic cycle in the earth's average temperature, as well as produces trends that move the global temperature up or down for tens or even hundreds of years [1, 2, 3]. However, it is less evident that short-term (weekly and monthly) changes in the global temperature are tied to solar activity whose short-time fluctuations would have the intermittent dynamics of solar flares. Traditional measures, such as cross-correlation functions, would not show the connection between short-time fluctuations in the global temperature and solar flare activity, because of the strong nonlinear hydrodynamic interactions across the earth's surface. For example, the hydrodynamic interaction of the atmosphere over land and water, would suppress any direct correlation between the intermittent sun's irradiance and the earth regions' short-time response.

This letter focuses on an alternate approach to establishing the connection between the sun's irradiance and the earth's temperature fluctuations. A link between the two phenomena is detected through a detailed scaling analysis of the time series for the earth's temperature and the time series for the solar flare frequency.

Considering a solar flare as an event, the time series for the number of solar flares has been interpreted as a waiting time distribution function between events. The solar flare waiting time distribution function is determined to be an inverse power-law probability density function (iplpdf) [4, 5, 6]. Such dynamical stochastic processes can be described by generalizations of random walks. A Lévy-flight, for example, is such a process with a diverging second moment. A Lévy-walk, on the other hand, visits the same spatial sites as does a Lévy-flight, but each step takes a finite time and the second moment is finite. The time necessary to complete a step in a Lévy-walk is specified by an inverse power-law waiting time distribution function, as first noticed by Shlesinger et al. 8]. It has been determined [4] that a Lévy-walk can describe the intermittent solar flare signal. Scafetta et al. 7, 9] established that, in general, the presence of a Lévy-walk process in a given time series can be detected by the joint use of two separate scaling techniques, the Diffusion Entropy Analysis (DEA) and Standard Deviation Analysis (SDA). We apply the same approach to the analysis of temperature data sets and compare them with the Lévywalk statistics induced by solar flare intermittency.

We study the earth's temperature anomalies both globally and locally, that is, North-South hemispheres and Land-Ocean regions, see Fig. 1. The technical term temperature anomalies denotes temperature departures from the 1961-1990 mean temperature value. These data are recognized in the geophysical community as among the most accurate data files for global air temperature, and global sea surface temperature (SST) [10]. The basic data set of global earth's temperature anomalies (HadCRUT) is a combination of Land air temperature anomalies 11 (CRUTEM1) and sea-surface temperature anomalies 12] on a $5^{\circ} \times 5^{\circ}$ grid-box basis. The merging of the two data sets is discussed in Parker et al. 12] and more recently in Jones et al. 13. The Land surface time series are calculated using data from the Global Historical Climatology Network (Version 2) and sea-surface temperature anomalies from the United Kingdom MOHSST data set and the NCEP Optimum Interpolated SSTs (Version2). The data that we analyze are mean monthly temperature anomalies downloaded from Climatic Research Unit, 


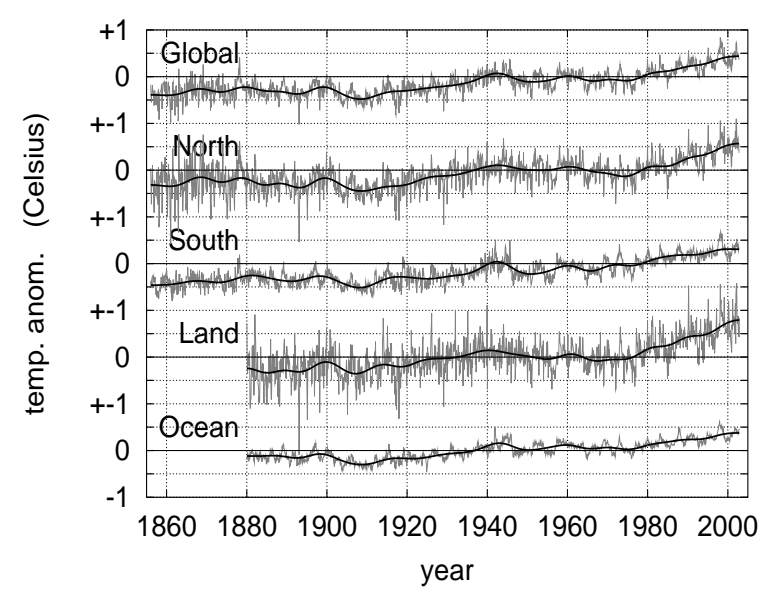

FIG. 1: Global and local Earth temperature anomalies in Celsius degree (years: 1856-2002).

UK, 14] (Global and North-South temperatures, 18562002) and from National Climatic Data Center, USA, [15] (Land-Ocean temperatures, 1880-2002).

The analysis of the above data sets is done with both DEA and SDA techniques. These methods are based on the prescription that the numbers in a time series $\left\{\xi_{i}\right\}$ are the fluctuations of a diffusion trajectory; see Refs. [4, 7, 9] for details. Therefore, we shift our attention from the time series $\left\{\xi_{i}\right\}$ to the probability density function (pdf) $p(x, t)$ of the corresponding diffusion process. Here $x$ denotes the variable collecting the fluctuations and is refered to as the diffusion variable. The scaling property of $p(x, t)$ takes the form

$$
p(x, t)=\frac{1}{t^{\delta}} F\left(\frac{x}{t^{\delta}}\right),
$$

where $\delta$ is the scaling exponent. The DEA [7] is based on the evaluation of the Shannon entropy, $S(t)$, using the pdf (11). If the scaling condition of Eq. (11) holds true, it is easy to prove that the entropy is

$$
S(t)=-\int_{-\infty}^{\infty} p(x, t) \ln [p(x, t)]=A+\delta \ln (t)
$$

where $A$ is a constant. The SDA [7], instead, is based on the evaluation of the standard deviation $D(t)$ using the same pdf (11) and yields to

$$
D(t)=\sqrt{\left\langle x^{2} ; t\right\rangle-\langle x ; t\rangle^{2}} \propto t^{H}
$$

where $H$ is the Hurst exponent [7, 16].

The Lévy-walk statistics is a particular form of anomalous diffusion obtained by generalizing the Central Limit Theorem 17. While all theoretical details can be found in Refs. [4, 7, 9] and in the enclosed references, here we show the properties of a Lévy-walk using a model that simulates solar flare intermittency. We generate an artificial sequence $\left\{\tau_{j}\right\}$ distributed according to an iplpdf,
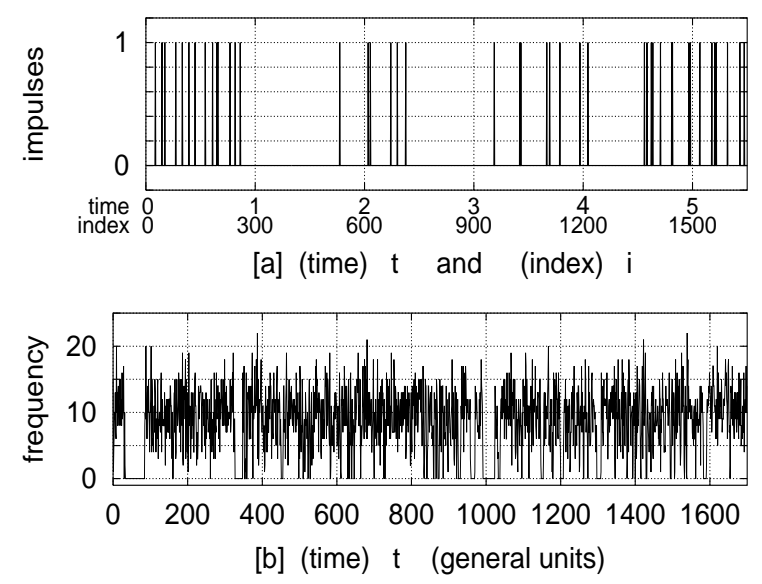

FIG. 2: Fig. 2a shows a computer-generated intermittent sequences of impulses. Fig. 2b shows the frequency of impulses for 1700 time units.

$$
\psi(\tau) \propto \frac{1}{(T+\tau)^{\mu}},
$$

where $T$ is a positive constant. In the computergenerated example, we use $T=90$ and $\mu=2.5$, that is, we use $2<\mu<3$, so as to violate the constraint of a finite variance on the central limit theorem that is necessary for obtaining Lévy-walk statistics. The sequence of waiting times $\left\{\tau_{j}\right\}$ is used to generate a time series $\left\{\xi_{i}\right\}$ given by $\xi_{i}=1$ only at the occurrence of the events, that is, for $i=\left[\sum_{j=1}^{m} \tau_{j}\right] ;[a]$ is the integer part of $a$. However, in the interval between two events, that is, for $\left[\sum_{j=1}^{m} \tau_{j}\right]<i<\left[\sum_{j=1}^{m+1} \tau_{j}\right]$, we assume $\xi_{i}=0$. As shown in Ref. [4, 7, 19] by using results of Refs. [17, 18, 19] the time series $\left\{\xi_{i}\right\}$ generates a diffusion process with a scaling exponent $\delta=1 /(\mu-1)$. Moreover, this particular approach to Lévy statistics yields a diffusion process with finite second moments, as in the Lévy-walk [8]. Consequently, it is possible to evaluate the scaling exponent of the standard deviation, $H$, via Eq. (3). Fig. 2a shows the first 1650 data points in the computer-generated sequence $\left\{\xi_{i}\right\}$, which correspond to the first 5.5 time units, of a 510,000 long data sequence $\left\{\xi_{i}\right\}$. In Fig. $2 \mathrm{~b}$ we show the sequence of frequencies $\left\{f_{t}\right\}$ of impulses for each time unit where we use 1 time unit $=300$ natural units of $\tau$.

The analysis is based on the comparison of the scaling exponents $\delta$ and $H$ measured by DEA and SDA respectively. It has been shown [7, 9] that if a time series is characterized by Gaussian statistics, the two scaling exponents are identical, that is, $\delta=H$. If, instead, the process under study is characterized by a Lévy-walk, the two scaling exponents $\delta$ and $H$ can be both related to the exponent $\mu$ of the waiting time $i p l p d f$ of the underling intermittent process generating the Lévy statistics. In the Lévy-walk case the scaling exponent $H$ is slightly larger than $\delta$ and the two exponents are related to $\mu$ and 


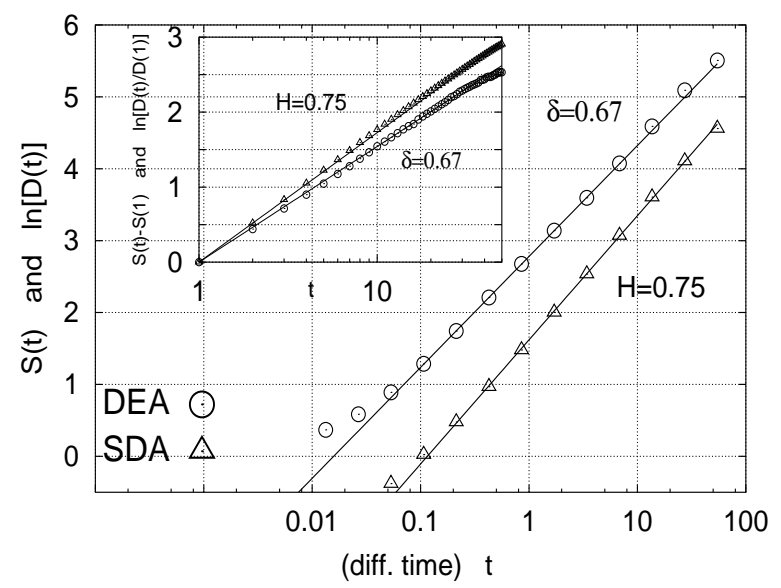

FIG. 3: DEA and SDA of the computer-generated Lévy-walk sequences shown in Figs. 2a and 2b. The two straight lines show the two theoretical scaling coefficients $\delta=0.67$ and $H=0.75$ for $\mu=2.5$.

one another via the following relation [7, 9]

$$
\delta=\frac{1}{3-2 H}=\frac{1}{\mu-1} .
$$

We refer to (5) as the Lévy-walk diffusion relation. The assessment of the property expressed by Eq. (5), that is, the comparison between the value of the scaling exponents $\delta$ and $H$ measured for the earth's temperature data sets and their relation with the scaling exponents $\delta$ and $\mu$ measured for the hard x-ray solar flares waiting time series [4], is used in this letter to verify the conjecture that the earth's global temperature inherits the intermittent Lévy-like nature of solar flares, within the temporal range from few weeks to few months.

In the computer-generated model we interpret the fluctuations in the frequency of the pulses shown in Fig. $2 \mathrm{~b}$ as inducing a similar memory pattern in the monthly mean global temperature fluctuations. Infact, a higher solar flare frequency implies a higher monthly solar activity. The DEA and SDA of the computer-generated data are shown in Fig. 3. These data are fit with Eqs. (2) and (3) yielding the two scaling coefficients $\delta=0.67$ and $H=0.75$. These coefficients are consistent with $\mu=2.5$ obtained by using Eq. (5); the known input value for this realization of the model. The large picture in Fig. 3 refers to the analysis of the original 510,000 data-point sequence $\left\{\xi_{i}\right\}$. The inset refers to the analysis of 1700 data-point frequency sequence $\left\{f_{t}\right\}$ shown in Fig. 2b, a length compatible with the length of the mean monthly temperature datasets here analyzed. The two graphs in Fig. 3 show very good agreement with Lévy-walk theory, that is, with Eq. (5) because both time series produce similar diffusion trajectories. In particular, the small inset in Fig. 3 shows the typical bifurcation associated to the two scaling laws that characterize Lévy-walk statistics and that the statistics generated by only $\mathrm{N}=1700$ frequency data points is rich enough to obtain a satisfac-
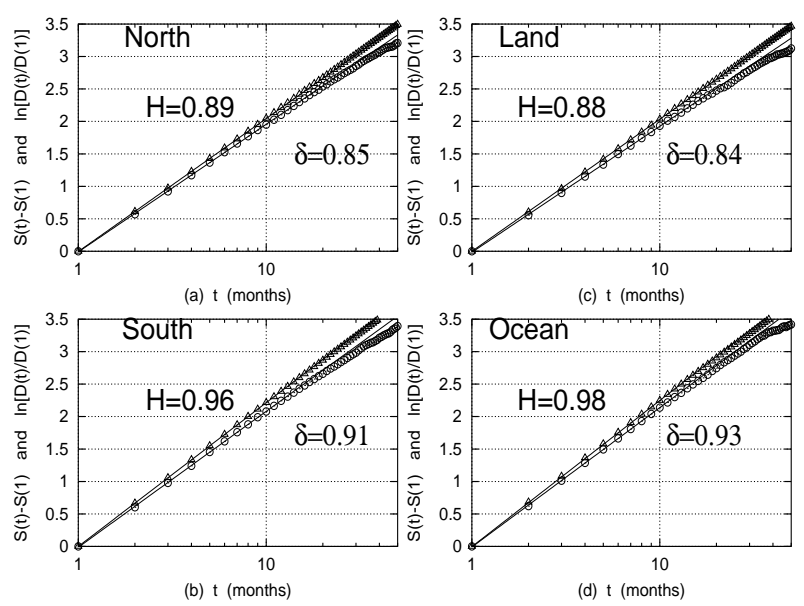

FIG. 4: DEA and SDA of the North-South hemisphere and Land-Ocean region temperature anomalies. The scaling exponent $H$ and $\delta$ are reported in the figure, the error is \pm 0.02 .

tory pdf with Lévy-walk scaling properties.

Fig. 4 shows the numerical results by applying DEA and SDA to the temperature fluctuation data of the four regions of the earth. On the ordinate axis we plot $\ln [D(t) / D(1)]$ and $S(t)-S(1)$; thus, all curves start from 0 . The scaling exponents $\delta$ and $H$ are obtained by fitting the first 10 points. This analysis for the Global temperature (not shown in the figure) yields $\delta=0.90 \pm 0.02$ and $H=0.95 \pm 0.02$. The high values of the exponents imply a strong persistence in the temperature fluctuations. Both scaling exponents $\delta$ and $H$ are slightly larger for the South hemisphere than for the North hemisphere and for the Ocean than for the Land. A reasonable explanation is that the Ocean is an almost stationary system with a very high effective heat capacity. The Land, instead, is subject to stronger random fluctuations (as Fig. 1 shows) due to a lower effective heat capacity and a higher morphological variability due, for example, to the presence of deserts, forests, mountains and valleys. Finally, we note that the standard deviation scaling exponents $H$ are larger than the diffusion entropy scaling exponents $\delta$ and seem to fulfill the Lévy-walk diffusion relation (5) within the accuracy of our statistical analysis.

The scaling behavior in the temperature anomalies can be related to the intermittency of the solar flare activity through the mechanism suggested by the model depicted in Figs. 2 and 3. In fact, the waiting time distribution of solar flares is characterized by an iplpdf of the type of Eq. (4) with $\mu=2.14 \pm 0.05$ [4]. This value of $\mu$ would imply a Lévy-walk with $\delta=0.88 \pm 0.02$ and $H=$ $0.93 \pm 0.02$ (5) for a shuffled dataset that would destroy any temporal correlation among flares and conserve only the Lévy component; see Ref. [4] about the effect of the shuffling on these data. We observe that the difference of the above two scaling exponent values gives $H-\delta=$ $0.05 \pm 0.02$, that is a value compatible with the difference between $H$ and $\delta$ measured for all the temperature data sets. In particular, $H-\delta=0.05 \pm 0.02$ for the Global 


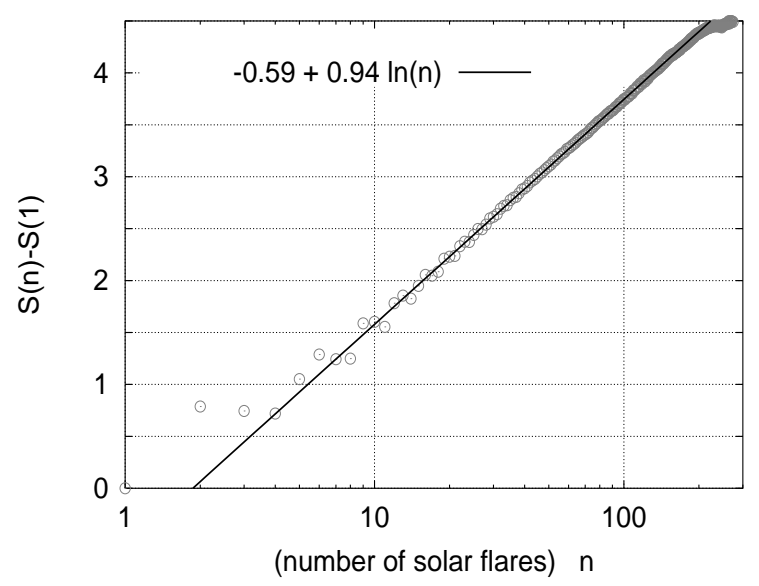

FIG. 5: DEA of the unshuffled waiting time of solar flares sequence during the period 1991-2000. The value of the scaling exponent is $\delta=0.94 \pm 0.02$.

temperature and for both South and Ocean regions and $H-\delta=0.04 \pm 0.02$ for North and Land regions.

To understand the actual values that we measure for $H$ and $\delta$ we first notice that the waiting time sequence of solar flares is weakly correlated [4]. Therefore, the effective value of $\delta$ for the original unshuffled sequence is slightly larger than that calculated via the waiting time iplpdf exponent $\mu$ for flares [4], $\delta=0.94 \pm 0.02$, as Fig. 5 shows. We observe that this value of $\delta$ is perfectly compatible with $\delta=0.93 \pm 0.02$ measured for the Ocean temperature. The Ocean, in fact, is the region of the earth that should best mirror solar activity because it is the largest and most homogeneous region of the earth with a very high effective heat capacity; properties that retain correlated events in the temperature. Also we observe that the occurrence of uncorrelated events would slightly reduce the Lévy-like memory and increase the Gaussianity of the data. This might explain why the scaling exponents $H$ and $\delta$ as well as their difference slightly decreases $(0.04$ against 0.05 ) between the North and Land regions. Finally, Fig. 5 shows that the scaling properties for the waiting time of solar flares last at least 200 consecutive flares and the waiting time for clusters of 200 solar flares may last for several months. The largest waiting time between two consecutive solar flares, during the period 1991-2000, is almost 3 months [4], and on average there are almost 60 solar flares per month. Therefore, the temporal resolution of these clusters is compatible with the temporal range of scaling of the temperature data sets that last at least 1 to almost 20 months, as Fig. 4 shows.

In conclusion, the affinity of the scaling exponents obtained through our analysis suggest that the earth's temperature anomalies inherits a Lévy-walk memory component from the intermittency of solar flares. We obtain reliable scaling properties within a short time interval, ranging from a few weeks to a few months. Morever, the joint use of SDA and DEA has proved to be very useful in detecting a significant Lévy component in the time series and, in general, it may suggest the presence of a link between a phenomenon under study and an intermittent process characterized by a waiting time iplpdf.

Acknowledgment: N.S. thanks the ARO for support under grant DAAG5598D0002.
[1] W. Soon, S. Baliunas, E. S. Posmentier, P. Okeke, New Astronomy 4 (8): 563-579 Mar 8 (2000); M. Sharma, Earth Planet sc Lett. 199 (3-4): 459-472 Jun 10 (2002); M. Lockwood, J. Geophys. Res-Space 106 (A8): 1602116038 Aug 1 (2001); W. Soon, E. Posmentier, S. Baliunas, Ann. Geophys. Atm. Hydr. and Space Sci. 18 (5): 583-588 May (2000); N. F. Arnold, T. R. Robinson, Ann. Geophys. Atm. Hydr. and Space Sci., 16 (1): 69-76 Jan (1998); S. Bravo, J. A. L. CruzAbeyro, Geophs. Res. Lett. 23 (6): 613-616 Mar 15 (1996); G. M. Brown, J. I. John, J. Atmos. Terr. Phys., 41 (1): 43-52 (1979); E. Friischristensen, K. Lassen, Science 254 (5032): 698-700 Nov 1 (1991).

[2] T. Baranyi, A. Ludmany, H. Coffey, Geophys. Res. Lett., 25 (13): 2269-2272 Jul 1 (1998); D. Shindell, D. Rind, N. Balachandran, J. Lean, P. Lonergan, Science 284 (5412): 305-308 APR 9 (1999); M. Lockwood, R. Stamper, M. N. Wild, Nature 399 (6735): 437-439 Jun 3 (1999).

[3] J. D. Fix, Astronomy, Journey to the Cosmic Frontier, 2nd edition, McGraw-Hill, NY USA, (2001).

[4] P. Grigolini, D. Leddon, N. Scafetta, Phys. Rev. E 65, 046203 ( 2002).

[5] G. Boffetta, V. Carbone, P. Giuliani, P. Veltri, and A. Vulpiani, Phys. Rev. Lett. 83, 4662 (1999).
[6] P. Giuliani, V. Carbone, P. Veltri, G. Boffetta, A. Vulpiani, Physica A 280, 75 (2000).

[7] N. Scafetta, P.Grigolini, Phys. Rev. E 66, 036130 (1-10) (2002).

[8] M. Shlesinger, B.J. West and J. Klafter, Phys. Rev. Lett. 58, 1100-1103 (1987).

[9] N. Scafetta, V. Latora, P. Grigolini, Phys. Rev. E 66, 031906 (2002).

[10] P.D. Jones, D.E. Parker, T.J. Osborn, and K.R. Briffa. In Trends: A Compendium of data on Global Change. Carbon Dioxide Information Analysis Center, Oak Ridge National Laboratory, U.S. Department of Energy, Oak Ridge, Tenn., U.S.A, 2001.

[11] P.D. Jones, Journal of Climate 7 (11):1794-1802, 1994.

[12] D.E. Parker, P.D. Jones, A. Bevan, and C.K. Folland, Journal of Geophysical Research 99:14373-14399, 1994.

[13] P.D. Jones, T.J. Osborn, K.R. Briffa, C.K. Folland, E.B. Horton, L.V. Alexander, D.E. Parker, and N.A. Rayner, Journal of Geophysical Research 106, 3371-3380, 2001.

[14] http://www.cru.uea.ac.uk: Climatic Research Unit, UK.

[15] http://lwf.ncdc.noaa.gov : National Climatic Data Center, USA.

[16] B.B. Mandelbrot, The Fractal Geometry of Nature, Freeman, New York, 1983. 
[17] B.V. Gnedenko and A.N. Kolomogorov, Limit Distributions for Sums of Random Variables, Addison-Wesley, Reading, MA, 1954.

[18] J. Klafter, M.F. Shlesinger, and G. Zumofen, Phys. To- day 49(2), 33 (1996); G. Zuomofen, J. Klafter, and M.F. Shlesinger, Lect. Notes Phys 519,15 (1998).

[19] W. Feller, Trans. Am. Math. Soc. 67, 981949. 